\title{
Análise da camada de fibras nervosas da retina em usuários crônicos do tabaco e álcool
}

\author{
Analysis of retinal nerve fiber layer in chronic tobacco and alcohol users
}

\author{
Rita de Cássia Ramos de Lima \\ Fernando Carvalho² \\ Silvio de Biase Siqueira Campos ${ }^{3}$ \\ Hellmann Dantas ${ }^{4}$
}

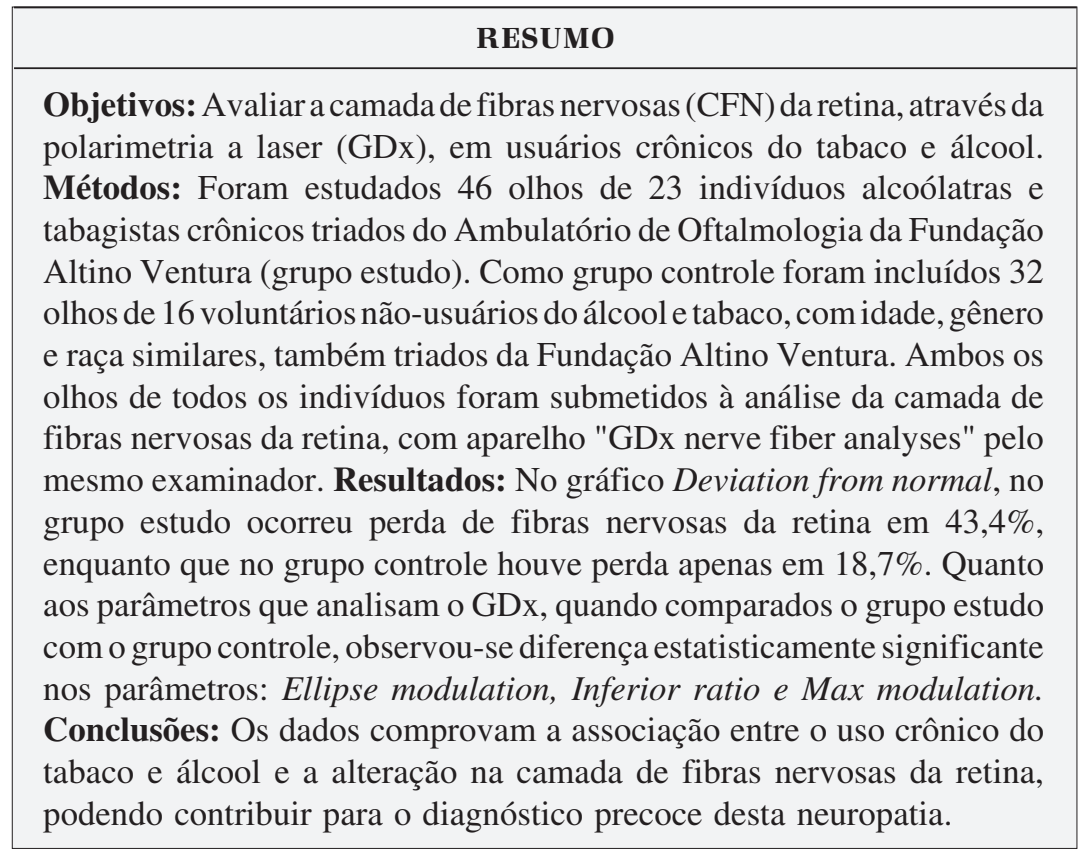

Descritores: Fibras nervosas; Retina; Tabagismo; Alcoolismo; Doenças do nervo óptico; Perimetria/métodos; Lasers/uso diagnóstico

\footnotetext{
${ }^{1}$ Fellow da Fundação Altino Ventura - Recife (PE) Brasil.

${ }^{2}$ Residente da Fundação Altino Ventura - Recife (PE) Brasil.

${ }^{3}$ Residente da Fundação Altino Ventura - Recife (PE) Brasil.

${ }^{4}$ Oftalmologista da Fundação Altino Ventura - Recife (PE) - Brasil.

Endereço para correspondência: FAV - Fundação Altino Ventura. Rua da Soledade, 170 - Boa Vista Recife (PE) CEP 50070-040

E-mail: fav@fundacaoaltinoventura.org.br

Recebido para publicação em 22.07.2005 Aprovação em 25.09.2005
}

\section{INTRODUÇÃO}

Alcoolismo tem sido definido quando há interferência do álcool no funcionamento da vida do indivíduo, associado com forte compulsão para utilizá-lo, acompanhado de tolerância aumentada ao etanol ou presença de sinais físicos secundários à abstinência. Em torno de $10 \%$ dos homens e 3 a $5 \%$ das mulheres são afetados pelo alcoolismo ${ }^{(1-2)}$.

Mudanças bioquímicas induzidas pelo álcool, podem diminuir a função da retina, fato que já foi detectado pelo eletrorretinograma ${ }^{(2)}$. Há relatos que a exposição crônica ao álcool também induz degenerações na camada de fotorreceptores da retina em ratos $^{(3)}$.

A polarimetria a laser (GDx) é um método objetivo e não invasivo, desenvolvido para avaliar quantitativamente a camada de fibras nervosas da retina $(\mathrm{CFN})$. Este fato acontece graças à birrefrigência que os axônios das células ganglionares apresentam à luz polarizada, que passa através da $\mathrm{CFN}$, sofrendo um retardo que vai ser proporcional à sua espessura ${ }^{(4-5)}$. O exame apresenta boa reprodutibilidade e índices de especifidade de $91,1 \% \mathrm{e}$ sensibilidade de $87,3 \%{ }^{(6)}$.

Face às alterações do sistema nervoso central induzidas pelo uso crônico do tabaco e álcool, o objetivo do presente trabalho foi avaliar a camada 
de fibras nervosas da retina, através do GDx, em indivíduos alcoolistas e tabagistas crônicos, visando estabelecer um diagnóstico mais precoce da neuropatia, já que esta condição pode ser de caráter reversível.

\section{MÉTODOS}

Realizou-se um estudo tipo caso controle em 23 pacientes (46 olhos) usuários crônicos do tabaco e do álcool (grupo estudo). Todos os pacientes do grupo de estudo, eram do gênero masculino, com idades variando entre 24 e 75 anos $(41,2 \pm 11,9$ anos). O consumo de álcool era de dois a cinco litros de álcool por dia ( $3,6 \pm 0,8$ litros/dia) em um período que variou de 10 a 40 anos (23,2 $\pm 7,9$ anos). Quanto ao tabaco, os pacientes fumavam entre uma e cinco carteiras por dia $(1,3 \pm 0,6$ carteiras/dia) em um período que variou de 14 a 36 anos ( $24,4 \pm 8,0$ anos). O grupo controle foi constituído por 16 indivíduos (32 olhos) não usuários do tabaco e álcool, com idade, gênero e raça similares.

Foram excluídos os voluntários que apresentassem pressão intra-ocular maior que $21 \mathrm{mmHg}$, presença de história familiar de glaucoma, acuidade visual igual ou pior do que 0,5 em ambos os olhos, escavação papilar maior do que $0,4 \mathrm{com}$ assimetria maior que 0,2 entre os dois olhos. Também foram excluídos os pacientes que tivessem outra doença ou cirurgia ocular prévia, alteração córneo-retiniana detectada ao exame clínico, erro refracional maior que dez dioptrias esféricas de hipermetropia ou miopia.

Os voluntários, pacientes e indivíduos, foram submetidos ao exame oftalmológico composto por acuidade visual, refração, reflexo fotomotor aferente e eferente, motilidade extrínseca, biomicroscopia do segmento anterior, aferição da pressão intra-ocular com tonômetro de Goldman e exame do fundo de olho. Os voluntários que satisfizeram os critérios foram submetidos à análise da camada de fibras nervosas da retina, utilizando a polarimetria a laser (GDx), pelo mesmo examinador. Foram obtidas duas imagens de cada olho sendo considerada a imagem média.

Os resultados das variáveis contínuas foram expressos por suas médias e desvio padrão. Para avaliação da possível diferença entre as médias, utilizou-se o teste "t" de Student para amostras não pareadas. As variáveis categóricas foram expressas por suas freqüências. Aceitou-se $\mathrm{p}<0,05$ para rejeição da hipótese de nulidade.

O estudo foi submetido à analise pelo comitê de ética da Fundação Altino Ventura, onde foi aprovado. Os pacientes foram triados no ambulatório da Fundação Altino Ventura e todos assinaram o termo de consentimento livre e esclarecido sobre o estudo.

\section{RESULTADOS}

No grupo estudo, no gráfico Deviation from normal, observou-se que houve perda moderada da camada de fibras nervo- sas (CFN) em nove olhos (19,6\%). Em seis olhos $(13,0 \%)$ houve perda leve e em cinco olhos $(10,8 \%)$ houve perda severa. A maioria dos pacientes $(56,6 \%)$ não apresentou perda da CFN neste gráfico. No grupo controle houve perda leve em cinco olhos $(15,6 \%)$ e moderada em um olho $(3,1 \%)$. A maioria dos olhos (81,3\%) também não apresentou nenhuma perda da CFN.

Entre os parâmetros que analisam as fibras nervosas no GDx, quando comparado o grupo estudo com o grupo controle, Ellipse modulation, Inferior ratio e Max modulation apresentaram diferença estatisticamente significante. A média e desvio padrão de cada parâmetro, nos dois grupos, estão descritos na tabela 1 .

\section{DISCUSSÃO}

O sistema nervoso é particularmente sensível aos efeitos do álcool. O nervo óptico tem sido estudado para detectar tais efeitos ${ }^{(7)}$.

O nervo óptico de ratos induzidos a ingesta crônica de álcool já foi analisado, sendo observadas alterações ultraestruturais importantes em todos os nervos ópticos, apesar de não ter sido encontrado alterações morfométricas ${ }^{(7)}$.

A ambliopia tabaco-álcool acomete $0,5 \%$ das pessoas que fazem uso abusivo dessas substâncias. É comprovado o envolvimento da carência nutricional na etiopatogenia desta doença, o que faz com que ela tenha um caráter reversível, tornando importante seu diagnóstico o mais precocemente possível ${ }^{(8-10)}$.

A camada de fibras nervosas da retina pode ser avaliada através do GDx, e neste estudo tentou-se mostrar possíveis alterações em usuários crônicos do tabaco-álcool, visando um diagnóstico mais precoce ${ }^{(4)}$. A perda de CFN mostrada no gráfico Deviation from normal sugere que o tabaco e álcool causa danos a esta camada.

Observou-se neste trabalho associação significante em alguns parâmetros que analisam o GDx em usuários crônicos do tabaco-álcool, reforçando a hipótese que o tabaco-álcool

\begin{tabular}{|c|c|c|c|c|c|}
\hline \multirow[t]{2}{*}{ Parâmetros } & \multicolumn{2}{|c|}{ Grupo estudo } & \multicolumn{2}{|c|}{ Grupo controle } & \multirow[b]{2}{*}{$\mathbf{p}$} \\
\hline & Média & DP & Média & DP & \\
\hline Symmetry & 0,93 & 0,15 & 0,97 & 0,07 & 0,30 \\
\hline Superior ratio & 2,35 & 0,55 & 2,36 & 0,42 & 0,30 \\
\hline Inferior ratio & 2,56 & 0,63 & 2,44 & 0,39 & 0,037 \\
\hline Superior/Nasal & 2,04 & 0,36 & 2,18 & 0,30 & 0,98 \\
\hline Max modulation & 1,68 & 0,58 & 1,54 & 0,41 & 0,025 \\
\hline Ellipse modulation & 2,99 & 0,94 & 2,76 & 0,53 & 0,012 \\
\hline The number & 19,39 & 17,78 & 12,5 & 4,96 & 0,82 \\
\hline Average thickness & 60,65 & 10,46 & 63,75 & 7,91 & 0,18 \\
\hline Ellipse average & 63,76 & 10,71 & 67,50 & 7,89 & 0,78 \\
\hline Superior average & 69,04 & 12,05 & 73,93 & 7,42 & 0,52 \\
\hline Inferior average & 76,93 & 13,37 & 82,34 & 10,41 & 0,12 \\
\hline Superior integral & 0,18 & 0,03 & 0,19 & 0,02 & 0,38 \\
\hline
\end{tabular}


altera a camada de fibras nervosas da retina, modificando o resultado do GDx.

Métodos para diagnóstico precoce desta neuropatia não são descritos na literatura. Então, existe a necessidade de exames complementares que possam vir a detectar alterações mais precocemente, numa fase reversível da neuropatia, com a suspensão do tabaco-álcool e tratamento adequado ${ }^{(9)}$. O prognóstico dos distúrbios visuais nesses pacientes, embora imprevisíveis, pode melhorar em cerca da metade dos mesmos com boa nutrição e suplementação de vitaminas, principalmente em fase precoce, todavia, em alguns pacientes a recuperação completa pode não ser alcançada ${ }^{(10)}$.

\section{CONCLUSÃO}

Vale ressaltar que o estudo da CFN da retina com GDx em usuários crônicos do tabaco-álcool é original na literatura, o que reforça a importância de novos estudos, com outros exames complementares como o campo visual e o potencial visual evocado, para que juntos possam vir a confirmar a hipótese testada da associação entre o uso crônico do tabaco-álcool e a perda de fibras nervosas da retina.

\section{ABSTRACT}

Purposes: To evaluate the retinal nerve fiber layer using GDx in chronic alcohol and tobacco users. Methods: Twenty-three chronic alcohol and tobacco users were studied (study group). As a control group, sixteen volunteers without use of alcohol and tobacco with similar characteristics (age, gender and race) were included. Both eyes were tested for analysis of the retinal nerve fiber layer with GDx. Results: There was alteration in the Deviation from normal graph with a loss of nerve fibers in $43.4 \%$ of patients in the study group. In the control group this alteration was observed in 14 eyes (18.7\%). As regards the parameters that allow comparison between the study and control groups there was a statistically significant difference regarding the following parameters: Ellipse modulation, Inferior ratio and Max modulation. Conclusions: The chronic use of tobacco and alcohol was associated with alteration of the nerve fiber layer. Thus, these results can contribute to the early diagnosis of nerve fiber layer loss in optic neuropathy.

Keywords: Nerve fibers; Retina; Smoking; Alcoholism; Optic nerve diseases; Perimetry/methods; Lasers/diagnostic use

\section{REFERÊNCIAS}

1. Isselbacher KJ, Braunwald E, Wilson JD, Martin JB, Fauci AS, Kasper DL, editores. Harrison Medicina Interna.13a ed. In: Schuckit MA. Álcool e alcoolismo. Mexico: Interamericana; 1994. p.2543-9.

2. Shimozono M, Townsend JC, Ilsen PF, Bright DC. Acute vision loss resulting from complications of ethanol abuse. J Am Optom Assoc. 1998;69(5): 293-303.

3. Pawlosky RJ, Bacher J, Salem N Jr. Ethanol consumption alters electroretinograms and depletes neural tissues of docosahexaenoic acid in rhesus monkeys: nutritional consequences of a low n-3 fatty acid diet. Alcohol Clin Exp Res. 2001;25(12):1758-65.

4. Luande-Pimentel R, Costa VP. Introdução à análise da camada de fibras nervosas da retina. In: Lauande-Pimentel R, Costa VP, editores. Um guia para interpretar o exame de polarimetria. Rio de Janeiro: Cultura Médica; 2001. p.9-16.

5. Hoyt WF, Frisen L, Newman NM. Fundoscopy of nerve fiber layer defects in glaucoma. Invest Ophthalmol. 1973;12(11):814-29.

6. Susanna Junior R, Takahashi WY, Nakamura NKF. Sensibilidade e especificidade da avaliação da camada de fibras nervosas da retina examinadas através da polarimetria a laser. Rev Bras Oftalmol. 1998;57(1):17-22.

7. Shiguematon AI, Schellini AS, Gregório EA, Pellizzon CH, Müller SS, Padovani CR. Análise morfométrica e ultra-estrutural do nervo óptico de ratos induzidas a ingestão do álcool. Arq Bras Oftalmol. 2003;66(3):321-4.

8. Celino AC, Celino M, Toscano J, Diniz JR, Ventura LO. Alterações oculares em pacientes consumidores de álcool. Rev Bras Oftalmol. 2001;60(3):229-32.

9. Dang CV. Tobacco-alcohol amblyopia: a proposed biochemical basis for pathogenesis. Med Hypotheses. 1981;7(11):1317-28.

10. Krumsiek J, Kruger C, Patzold U. Tobacco-alcohol amblyopia neuro-ophtalmologial findings and clinical course. Acta Neurol Scand. 1985;72(2):180-7.

\section{PBO ELETROANICO \\ A versão eletrônica dos Arquivos Brasileiros de Oftalmologia com textos completos está disponível em:}

- ABD - Arquivos Brasileiros de Oftalmologia - http://www.abonet.com.br

- 5ciELם - Scientific Electronic Library Online - http://www.scielo.org

- Free Medical Journals - http://www.freemedicaljournals.com

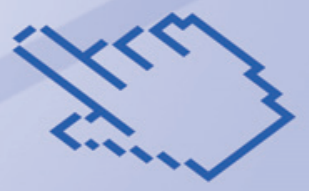

